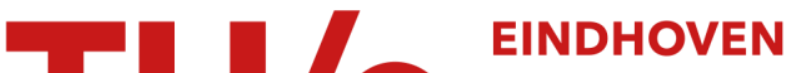

\section{Fetal movement quantification by fetal vectrocardiography: a preliminary study.}

\section{Citation for published version (APA):}

Vullings, R., Peters, C. H. L., Mischi, M., Oei, S. G., \& Bergmans, J. W. M. (2010). Fetal movement quantification by fetal vectrocardiography: a preliminary study. In Proceedings on the 30th Annual International Conference of the IEEE Engineering in Medicine and Biology Society 2008, EMBS 2008, 20-25 August 2008, Vancouver, British Columbia (pp. 1056-1059). Institute of Electrical and Electronics Engineers.

https://doi.org/10.1109/IEMBS.2008.4649341

DOI:

10.1109/IEMBS.2008.4649341

Document status and date:

Published: 01/01/2010

\section{Document Version:}

Publisher's PDF, also known as Version of Record (includes final page, issue and volume numbers)

\section{Please check the document version of this publication:}

- A submitted manuscript is the version of the article upon submission and before peer-review. There can be important differences between the submitted version and the official published version of record. People interested in the research are advised to contact the author for the final version of the publication, or visit the $\mathrm{DOI}$ to the publisher's website.

- The final author version and the galley proof are versions of the publication after peer review.

- The final published version features the final layout of the paper including the volume, issue and page numbers.

Link to publication

\section{General rights}

Copyright and moral rights for the publications made accessible in the public portal are retained by the authors and/or other copyright owners and it is a condition of accessing publications that users recognise and abide by the legal requirements associated with these rights.

- Users may download and print one copy of any publication from the public portal for the purpose of private study or research.

- You may not further distribute the material or use it for any profit-making activity or commercial gain

- You may freely distribute the URL identifying the publication in the public portal.

If the publication is distributed under the terms of Article 25fa of the Dutch Copyright Act, indicated by the "Taverne" license above, please follow below link for the End User Agreement:

www.tue.nl/taverne

Take down policy

If you believe that this document breaches copyright please contact us at:

openaccess@tue.nl

providing details and we will investigate your claim. 


\title{
Fetal movement quantification by fetal vectorcardiography: a preliminary study
}

\author{
R Vullings, CHL Peters, M Mischi, SG Oei, and JWM Bergmans
}

\begin{abstract}
Fetal movement is a valuable source of information to monitor the neurological development of the fetus and assess fetal health. Currently, fetal movement can be assessed by the mother or detected by analysis of ultrasound images. Long-term monitoring of movement is complicated with both these methods as maternal self-assessment has a relatively poor sensitivity and specificity and automatic analysis of ultrasound images is not available. Moreover, ultrasound transducers transmit energy into the body, potentially endangering fetal health. In this paper, an alternative method for fetal movement monitoring is presented. This method operates by estimating and analyzing the fetal vectorcardiogram (VCG) from non-invasive recordings on the maternal abdomen. The determined fetal movement is compared with that assessed from a simultaneously performed ultrasound recording; the results of the presented method are consistent with the ultrasound images. In addition, the presented method enables quantification of the rotation angles by means of analysis of the rotation matrix between consecutive fetal VCGs, providing a tool for long-term monitoring of fetal movement with increased specificity.
\end{abstract}

\section{INTRODUCTION}

During pregnancy the fetal health condition is generally monitored by assessing fetal heart rate (FHR) variability and fetal movements. The degree of FHR variability in combination with fetal movement [1] provides indirect information on the physiological state of the fetus, e.g. when the fetus is asleep the FHR variability and motility is expected to be smaller than when the fetus is awake and active. The frequency with which transitions between these physiological states occur is used by physicians to monitor the development of the fetus with progressing pregnancy [2].

In other words, both the FHR variability and fetal movement are indirect parameters to assess the physiological state of the fetus. In general, the FHR and fetal motility are assessed by means of Doppler ultrasound and echography, respectively, but fetal movement can also be established through subjective assessment of the mother.

Assessment by the mother has relatively poor sensitivity and specificity [3] and can cause unnecessary anxieties when the mother notices a decrease in fetal movement. In addition, ultrasound transducers transmit energy into the body, potentially endangering fetal health, and no automated analysis of the ultrasonic images is commercially available.

This work was supported by the Dutch technology foundation STW

R Vullings, M Mischi, and JWM Bergmans are with the Eindhoven University of Technology, Department of Electrical Engineering, Eindhoven, the Netherlands r. vullingsetue.nl

CHL Peters is with the Amphia Hospital, Department of Clinical Physics, Breda, the Netherlands

SG Oei is with the Máxima Medical Center, Department of Gynecology and Obstetrics, Veldhoven, the Netherlands
Hence, accurate long-term monitoring of fetal movement with existing methods is difficult. From this it is clear that any other method for monitoring fetal movement that is objective and enables long-term monitoring can be highly valuable.

In this paper, we propose the analysis of the fetal vectorcardiogram (VCG) as a method for monitoring fetal movements. Comparison of consecutive fetal VCGs provides information on the rotation of the fetal heart and consequently on the rotation of the fetal thorax. Accordingly, a disadvantage of this method is that only movement of the fetal thorax can be detected and no movement of the extremities. However, the method is capable of long-term monitoring and hence has added value to the existing methods, in particular as the specificity is expected to be significantly larger than for assessment of fetal movement by the mother. Moreover, the assessment of fetal movement through analysis of the fetal VCG provides simultaneous information on the FHR variability, requiring no additional measurements. As a result, the recording and analysis of the fetal VCG provides the two main parameters to assess the physiological state of the fetus at once.

The fetal VCG can be recorded from the maternal abdominal wall using contact electrodes. The signals recorded by these electrodes contain a mixture of electrophysiological signals from both mother and fetus and noise. One of the signals originating from the fetus is the fetal electrocardiogram (ECG). The spatial information of the fetal ECG, obtained by using multiple electrodes spread over the maternal abdomen, can be combined to produce the fetal VCG.

As mentioned previously, consecutive VCGs can be compared to provide information on the rotation of the fetal heart. In general, this rotation can be quantified by calculating the rotation matrix between consecutive VCGs. To facilitate long-term monitoring, this rotation matrix can be analyzed to produce three individual rotation angles that describe the rotation in three dimensional space.

The method is evaluated by comparing the detected fetal movement to fetal movement determined from a simultaneously performed ultrasound recordings.

\section{Methodology}

\section{A. Fetal ECG}

The recordings are performed at the Máxima Medical Center, Veldhoven, the Netherlands, using 8 abdominal electrodes, a common reference electrode, and an active ground electrode (Fig. 1). The choice for the electrode configuration is based on the VCG requirements and discussed 


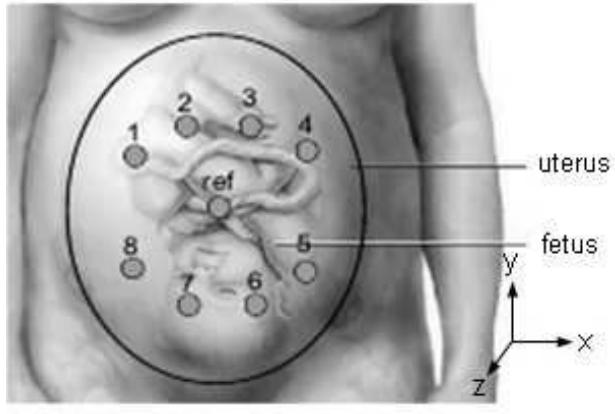

Fig. 1. Electrode configuration. The grounding electrode is not shown in this figure.

in Section II-B. All the cables are actively shielded to minimize the external interferences. The signals measured by these electrodes are digitized at $1 \mathrm{kHz}$ sampling rate using an MPAQ (Maastricht Instruments BV, the Netherlands), a programmable amplifier with 20 bit resolution. For this preliminary study, a single, healthy patient was used with gestational age of 24 weeks.

As mentioned previously, the signals recorded by the abdominal electrode contain a mixture of electrophysiological sources and noise. The main interference is the maternal ECG, which is removed by means of dynamically segmenting the maternal ECG complexes and subsequently estimating these maternal ECG segments by means of a linear prediction method as proposed in [4]. The resulting signals consist of the fetal ECG, electromyographic noise, and other noise sources, e.g. the interference from the powerline grid.

The signal to noise ratio (SNR) of the fetal ECG is increased by averaging 30 consecutive ECG complexes, all synchronized on the QRS-complex. Reason for using 30 complexes is that it ensures maximum increase in the fetal ECG SNR, while morphological changes in the ECG are not suppressed due to averaging [5]. In general, the healthy FHR is less regular than the adult heart rate. In particular, in fetuses of gestational ages of 24 weeks or less the nervous system has not developed completely yet, which can be reflected in the heart rate by ectopic beats [6]. In addition, artifacts are expected to occur in the recordings, both effects resulting in decreased SNR of the average fetal ECG. By applying a correlation scheme and FHR analysis, fetal ECG complexes that originate from ectopic beats or suffer from artifacts are excluded from the averaging, resulting in an SNR improvement. This correlation scheme rejects ECG complexes that exhibit a relatively low cross-correlation with other complexes, whereas the FHR analysis detects instantaneous heart intervals that deviate significantly from preceding intervals. Fig. 2 illustrates the removal of the maternal ECG and the averaging of the fetal ECG complexes.

\section{B. Fetal VCG}

The fetal ECG can be determined from the fetal VCG by means of the Dower matrix [7]. In fact, the fetal ECG is a projection of the fetal VCG on the vector that describes the bipolar ECG, e.g. the fetal ECG complex of Fig. 2c is
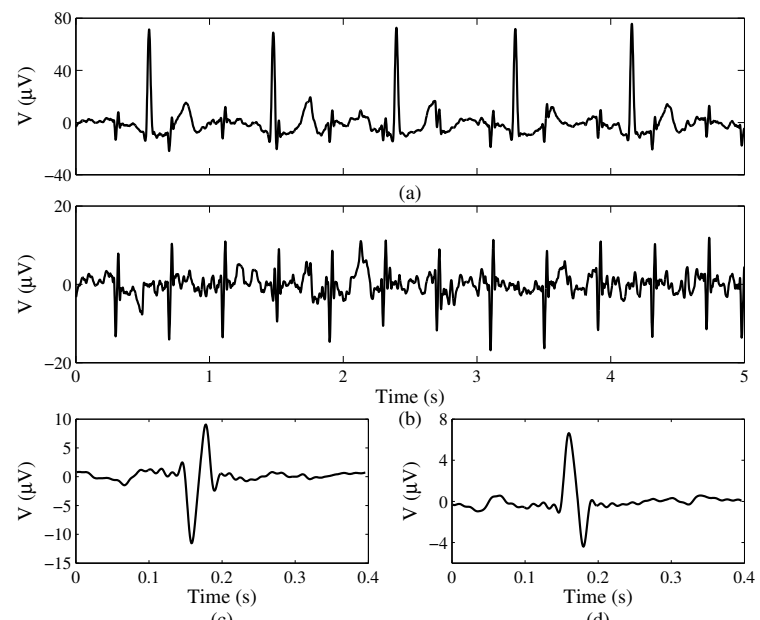

Fig. 2. Average fetal ECG calculation, with (a) part of the abdominal signal recorded from electrode 4 (Fig. 1), (b) the same signal after removal of the maternal ECG, and (c) and (d) the average fetal ECG complexes for electrodes 4 and 8, respectively (Fig. 1).

the projection of the VCG on the vector from the reference electrode to electrode 4 (Fig. 1). Hence,

$$
V=D S,
$$

with $V$ a $[N \times M]$ matrix containing the $N$ fetal ECG leads, with $M$ the length of the ECG complex, $S$ the $[3 \times M]$ matrix describing the fetal VCG, and $D$ the $[N \times 3]$ Dower matrix. Each row of this Dower matrix contains the vectorized electrode positions. This implies that the conduction of the electrical activity of the fetal heart to the maternal abdominal surface is assumed to have identical attenuation for all electrodes. In practice, until 28 weeks of gestation the conductive properties of the tissue between fetus and abdomen can be assumed uniform [8]. After this time, the vernix caseosa develops, changing these conductive proporties. In addition, considering the electrode configuration of Fig. 1 and assuming the fetal heart to be near the reference electrode, the distance to the fetal heart is approximately equal for all electrodes. Consequently, the attenuation can be assumed to be identical for all electrodes.

For gestational ages above 28 weeks or for situations in which the distance between electrodes and the fetal heart differs, the VCG is expected to be distorted. However, relative changes in this distorted VCG can still be related to fetal movement, although quantification of this movement will be more difficult or even impossible.

Therefore, with the model of Eq. (1) and the fetal ECG recorded from the maternal abdomen, the fetal VCG can be determined:

$$
S=D^{\dagger} V
$$

with $D^{\dagger}$ the Moore-Penrose inverse of the Dower matrix [9]. Fig. 3 shows the VCG corresponding to the fetal ECG complexes of Fig. 2. 


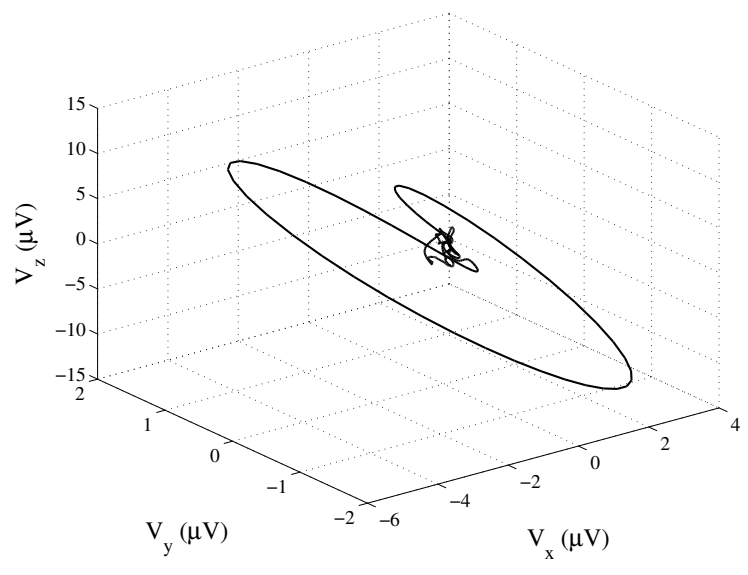

Fig. 3. Plot of the fetal VCG. The directions of the axes are indicated in the electrode configuration of Fig. 1.

\section{Fetal movement}

We assume $S_{\tau_{1}}$ the VCG at time $t=\tau_{1}$ and $S_{\tau_{2}}$ the VCG at time $t=\tau_{2}$, one fetal heart beat after $\tau_{1}$. The matrix describing the rotation between $S_{\tau_{1}}$ and $S_{\tau_{2}}$ is defined as $R$ :

$$
S_{\tau_{2}}=R S_{\tau_{1}}
$$

As consecutive heart beats have different lengths, the number of samples in consecutive VCGs is different. In order to determine the rotation matrix $R$, the VCG at time $\tau_{2}$ is resampled to match the length of $S_{\tau_{1}}$. In addition, consecutive VCGs are not identical in shape and hence $R$ must describe not only a rotation, but also a transformation. Consequently, quantitative assessment of the rotation trajectory from $R$ is complicated.

This rotation can, however, be estimated using an iterative procedure, minimizing the mean squared error (MSE) between $\tilde{S}_{\tau_{2}}$ and $\hat{R} S_{\tau_{1}}$, with $\tilde{S}_{\tau_{2}}$ the resampled VCG $S_{\tau_{2}}$ and $\hat{R}$ the estimated rotation matrix, a function of the rotation angle vector $(\phi, \theta, \psi)$.

In fact, the rotation matrix can be described by three rotation (Euler [10]) angles $\phi, \theta$, and $\psi$ :

$$
\begin{aligned}
& R=\left(\begin{array}{ccc}
1 & 0 & 0 \\
0 & \cos \psi & \sin \psi \\
0 & -\sin \psi & \cos \psi
\end{array}\right)\left(\begin{array}{ccc}
\cos \theta & 0 & \sin \theta \\
0 & 1 & 0 \\
-\sin \theta & 0 & \cos \theta
\end{array}\right) \cdots \\
& \ldots\left(\begin{array}{ccc}
\cos \phi & \sin \phi & 0 \\
-\sin \phi & \cos \phi & 0 \\
0 & 0 & 1
\end{array}\right)
\end{aligned}
$$

\section{Ultrasonic fetal movement assessment}

The ultrasonic recordings are performed using an Aloka SSD1100 (Aloka, Japan) ultrasound console with a $3.5 \mathrm{MHz}$ abdominal probe. This probe is positioned between the electrodes (Fig. 1) on the maternal abdomen and the images are stored using a frame grabber card.

For the single recording used in this study, fetal movement is not assessed by maternal perception next to the ultrasound analysis. Reason for this is that it is difficult for the mother to distinguish between movement of fetal extremities or fetal thorax and hence comparison between movements assessed by the mother and movements determined from VCG analysis is difficult to make.

\section{RESULTS}

In Fig. 4 the fetal VCG and the corresponding ultrasound image are shown for five different points in time. For clarity, only the QRS-loop of the fetal VCG is depicted. Reason for excluding the other segments of the VCG from Fig. 4 is the relatively low SNR of these segments (see Fig. 3).

From Fig. 4 it can be seen that the fetus is significantly rotating after $t=231 \mathrm{~s}$. After this time, the fetus turns its back more to the ultrasound probe, which is reflected in both the VCG and the ultrasound image (Fig. 4(e)).

Fig. 5 shows the trajectories of the rotation angles $\phi, \theta$, and $\psi$. In contrast to Fig. 4, Fig. 5 also shows significant fetal movement around $t=120 \mathrm{~s}$. Consequently, we can speculate that analysis of the rotation angles trajectories has additional value over visual analysis of the fetal VCG alone. However, as it is difficult to make conclusive statements on fetal rotation in the ultrasound images around $t=120 \mathrm{~s}$, this additional value needs to be confirmed by performing a larger number of recordings.

\section{DISCUSSION AND CONCLUSIONS}

In this paper a method is presented to monitor fetal movement by means of analysis of the fetal VCG. Visual comparison of the VCG shows that fetal movement is consistent with movement that is assessed from simultaneously performed echography. In addition, by estimating the rotation matrix between consecutive VCGs and analyzing the rotation angles trajectories, quantitative information on fetal movement can be obtained.

However, drawback of the presented method is that only movement of the fetal thorax can be detected; not of fetal extremities. In addition, only rotational movement can be detected. To assess translational fetal movement, other methods like time-delay analysis need to be adopted. Finally, inaccurate electrode positions, e.g. due to respirational movement of the maternal abdomen, or non-uniform conduction of the signals, e.g. due to development of the vernix caseosa, cause distortion of the VCG.

Movement of the electrodes due to maternal respiration is suppressed to a significant extent by averaging of the fetal ECG complexes. Distortion of the VCG due to non-uniform conduction complicates absolute assessment of the VCG. However, for fetal movement detection, only relative changes of the VCG are relevant. As the conductive properties of the maternal and fetal tissues vary only gradually, the changes in conduction are negligible for movement detection.

In spite of these flaws, the presented method provides a useful tool for long-term, quantitative monitoring of fetal movement. In addition, this method is expected to exhibit a larger specificity than maternal assessment and hence can have significant value for monitoring pregnancies at risk. However, conclusive statements about the value of this 

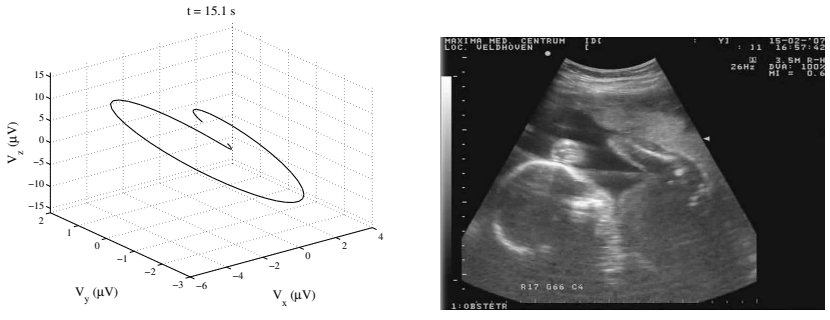

(a)
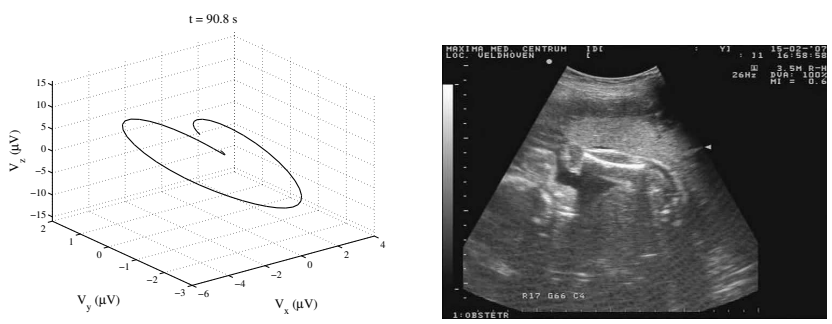

(b)
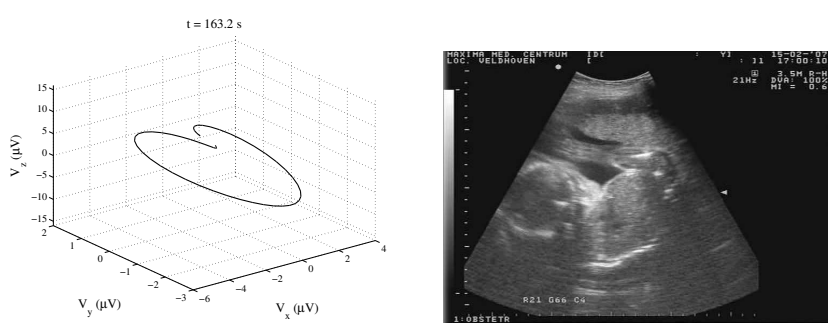

(c)
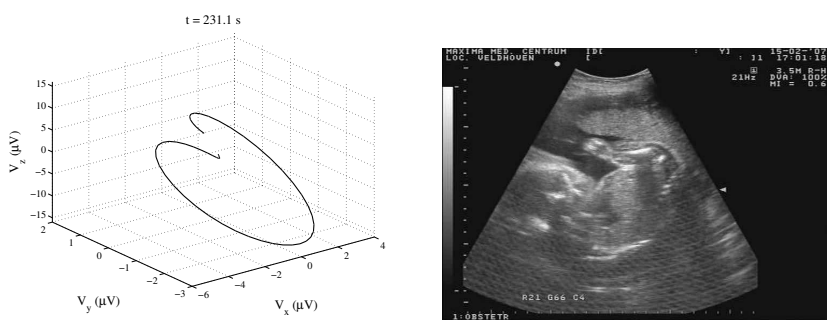

(d)
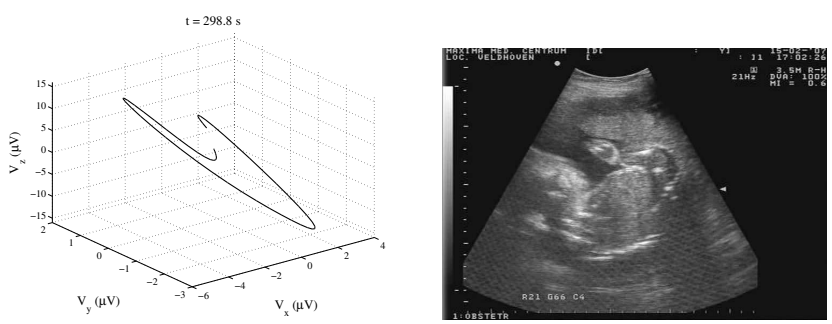

(e)

Fig. 4. QRS-loop of the fetal VCG and the corresponding ultrasound images at five points in time: (a) $t=15 \mathrm{~s}$, (b) $t=91 \mathrm{~s}$, (c) $t=163 \mathrm{~s}$, (d) $t=231 \mathrm{~s}$, and (e) $t=299 \mathrm{~s}$. The directions of the axes are indicated in Fig. 1.

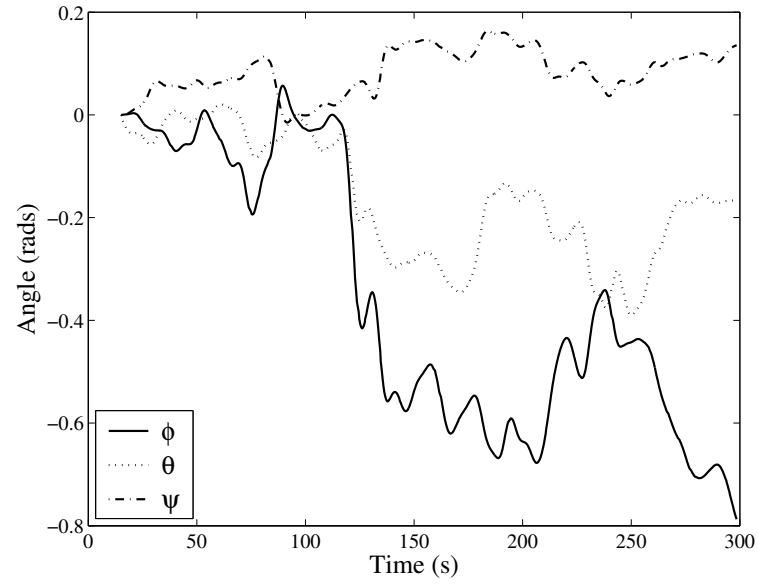

Fig. 5. Trajectories of the rotation Euler angles $\phi, \theta$, and $\psi$. These trajectories are obtained by calculating the cumulative sums of the instantaneous rotation angles.

method can only be made after analysis of more recordings, in which abdominal fetal ECG recordings are combined with both ultrasound and maternal assessment.

Future studies will include the application of sequential Bayesian analysis [11]. This method could improve assessment of fetal movement by exploiting periodicity and spatial correlation of the VCG, reducing the effects of low SNR and variability in the fetal ECG shape.

\section{REFERENCES}

[1] J.I.P de Vries, G.H.A. Visser, and H.F.R. Prechtl, "The emergence of fetal behaviour. I. Qualitative aspects", Early Human Development, vol. 7, 1982, pp 301-322.

[2] J. ten Hof and L.J.M. Nijhuis, "Development of fetal heart rate and behaviour", PhD thesis, University of Utrecht, the Netherlands, 1998.

[3] W. Schmidt, I. Cseh, K. Hara, and F. Kubli, "Maternal perception of fetal movements and real-time ultrasound findings" J Perinat Med, vol. 12, 1984, pp 313-318.

[4] R. Vullings, C. Peters, M. Mischi, R. Sluijter, G. Oei, and J. Bergmans, "Artifact reduction in maternal abdominal ECG recordings for fetal ECG estimation", in IEEE EMBS Proc. on the 29th Annual Internation Conference, Lyon, France, Aug. 23-26, 2007, pp. 43-46.

[5] A.K. Sundström, D. Rosén, and K.G. Rosén, Fetal Surveillance, Neovente Medical AB, Göteborg, Sweden, Sep. 2002.

[6] P. van Leeuwen, B. Hailer, W. Bader, J. Geissler, E. Trowitzsch, and D.H.W. Grönemeyer, "Magnetocardiography in the diagnosis of fetal arrhythmia", BJOG, vol. 106, Nov. 1999, pp 1200-1208.

[7] G.E. Dower, H.B. Machado, and J.A. Osborne, "On deriving the electrocardiogram from vectorcardiographic leads", Clin. Cardiol., vol. 3, 1980, pp 87-95.

[8] T.F. Oostendorp, A. van Oosterom, and H.W. Jongsma, "The fetal ECG throughout the second half of gestation", Clin. Phys. Physiol. Meas., vol. 10, 1989, pp 147-160.

[9] L. Edenbrandt and O. Pahlm, "Vectorcardiogram synthesized from a 12-lead ECG: superiority of the inverse Dower matrix", J Electrocardiol, vol. 21, no. 4, Nov. 1988,pp 361 - 367.

[10] E.W. Weisstein, "Euler angles", from Mathworld - A Wolfram Web Resource, http://mathworld.wolfram.com/EulerAngles.html.

[11] J. DeFreitas, M. Niranjan and A. Gee, "Hierarchical Bayesian-Kalman models for regularisation and ARD in sequential learning", Technical report, Department of Engineering, Cambridge University, 1998. 